\title{
Universiteit
}

Leiden

The Netherlands

\section{Motor imagery: A window into the mechanisms and alterations of the motor system.}

Lange, F.P. de; Roelofs, K.; Toni, I.

\section{Citation}

Lange, F. P. de, Roelofs, K., \& Toni, I. (2008). Motor imagery: A window into the mechanisms and alterations of the motor system. Cortex, 44, 494-506. Retrieved from https://hdl.handle.net/1887/14305

Version: $\quad$ Not Applicable (or Unknown)

License: $\quad$ Leiden University Non-exclusive license

Downloaded from: https://hdl.handle.net/1887/14305

Note: To cite this publication please use the final published version (if applicable). 


\title{
Special issue: Original article
}

\section{Motor imagery: A window into the mechanisms and alterations of the motor system}

\author{
Floris P. de Lange $e^{a, *}$, Karin Roelofs ${ }^{b}$ and Ivan Toni ${ }^{a, c}$ \\ ${ }^{a}$ F.C. Donders Centre for Cognitive Neuroimaging, Radboud University Nijmegen, Netherlands \\ ${ }^{\mathrm{b}}$ Department of Clinical and Health Psychology, University of Leiden, Netherlands \\ ${ }^{c}$ Nijmegen Institute for Cognition and Information, Radboud University Nijmegen, Netherlands
}

\section{A R T I C L E I N F O}

Article history:

Received 6 March 2007

Reviewed 15 May 2007

Revised 5 July 2007

Accepted 18 September 2007

Published online

Keywords:

Implicit motor imagery

Explicit motor imagery

Conversion paralysis

fMRI

Mental rotation

\begin{abstract}
A B S T R A C T
Motor imagery is a widely used paradigm for the study of cognitive aspects of action control, both in the healthy and the pathological brain. In this paper we review how motor imagery research has advanced our knowledge of behavioral and neural aspects of action control, both in healthy subjects and clinical populations. Furthermore, we will illustrate how motor imagery can provide new insights in a poorly understood psychopathological condition: conversion paralysis (CP). We measured behavioral and cerebral responses with functional magnetic resonance imaging ( $\mathrm{fMRI}$ ) in seven $\mathrm{CP}$ patients with a lateralized paresis of the arm as they imagined moving the affected or the unaffected hand. Imagined actions were either implicitly induced by the task requirements, or explicitly instructed through verbal instructions. We previously showed that implicitly induced motor imagery of the affected limb leads to larger ventromedial prefrontal responses compared to motor imagery of the unaffected limb. We interpreted this effect in terms of greater self-monitoring of actions during motor imagery of the affected limb. Here, we report new data in support of this interpretation: inducing self-monitoring of actions of both the affected and the unaffected limb (by means of explicitly cued motor imagery) abolishes the activation difference between the affected and the unaffected hand in the ventromedial prefrontal cortex. Our results show that although implicit and explicit motor imagery both entail motor simulations, they differ in terms of the amount of action monitoring they induce. The increased self-monitoring evoked by explicit motor imagery can have profound cerebral consequences in a psychopathological condition.
\end{abstract}

๑ 2007 Elsevier Masson Srl. All rights reserved.

\section{Introduction}

Motor imagery is a familiar aspect of most people's everyday experience. It is important for learning complex motor skills like sports (Murphy, 1994), as well as re-learning motor skills in neurological populations (Dijkerman et al., 2004; Lotze et al., 2006). The potential of motor imagery in clinical applications is broad, ranging from Brain-Computer interfacing (Pfurtscheller and Neuper, 2006) to diagnosis of vegetative state in non-communicative brain-injured patients (Owen et al., 2006).

\footnotetext{
${ }^{*}$ Corresponding author. F.C. Donders Centre for Cognitive Neuroimaging, Radboud University Nijmegen, Kapittelweg 29,6500 HB Nijmegen, Netherlands.

E-mail address: florisdelange@gmail.com (F.P. de Lange).

0010-9452/\$ - see front matter @ 2007 Elsevier Masson Srl. All rights reserved.
} doi:10.1016/j.cortex.2007.09.002 
Numerous studies have addressed behavioral and cerebral correlates of motor imagery, and its relationship with actual execution and motor planning [reviewed in Jeannerod, 2006]. Owing to this link, motor imagery paradigms have been extensively used as a tool to gain insight in the action system of both healthy and diseased populations. An important asset of motor imagery is that it allows one to investigate internal dynamics of motor control like planning and preparation, while avoiding sensory and motor confounds related to motor execution. This feature is especially important when studying motor impairments in clinical populations. In neuropsychiatric or neurological syndromes like hemiplegia, dystonia, Parkinson's disease, or a (conversive) limb paralysis, motor execution is impaired or even absent. In these cases, the (in)ability of imagining to carry out actions, and its cerebral correlates, can be used to establish at what level impairments in the action system are manifest.

The goal of this paper is threefold. Firstly, we assess how motor imagery research has advanced the knowledge of action control, reviewing behavioral and neuroimaging studies in healthy subjects. Secondly, we review the use of motor imagery in clinical populations, and its usefulness for scientific and diagnostic purposes.

Thirdly, we illustrate the use of motor imagery in a psychopathological condition: conversion paralysis (CP). In this context, we present new behavioral and neuroimaging data dealing with the cerebral implementation of imagined actions in the affected and non-affected arm, showing how manipulating the degree of action monitoring of the patient influences the imagery process.

\subsection{Motor imagery paradigms}

Motor imagery paradigms come in many flavours. One variable that differs between studies is the effector(s) that are used in the imagined action (e.g., hand, foot, mouth). Also, the complexity of the action to be imagined can vary widely, ranging from simple finger tapping (Hanakawa et al., 2003) to walking (Bakker et al., 2007; Stevens, 2005) or playing tennis (Owen et al., 2006). A further important distinction can be made between tasks that explicitly ask subjects to engage in motor imagery and tasks that elicit imagined actions in an implicit fashion (Jeannerod and Frak, 1999).

During explicit imagery tasks subjects are simply asked to imagine moving their effector in a particular manner [e.g., "Imagine making repetitive brisk flexion/extension movements of the fingers", Ehrsson et al., 2003]. Implicit imagery tasks on the other hand usually employ a task that is tangential to imagery of actions [e.g., "Is the stimulus you are looking at a left or right hand", Parsons, 1987; Sekiyama, 1982], and infer the motoric nature of the processes involved in solving the task from the behavior of the subjects. Conceptually, implicit and explicit imagery tasks differ in terms of how vulnerable they are to criticisms of cognitive penetrability (Pylyshyn, 2002). When subjects are explicitly asked to imagine a movement, say imagining to run from $A$ to $B$, they may use tacit knowledge about the time it takes to run from $A$ to $B$ to guide their performance, out of a desire to comply with the experimenter. This criticism applies less to implicit motor imagery tasks. In this case, subjects are not asked to engage in imagery, but to solve a tangential task (e.g., judge the laterality of a hand), and subjects are often not aware of the crucial experimental variables. In these cases, the visual or motor nature of the imagery process is inferred from behavioral and/or neural performance.

Another important dimension on which imagery paradigms differ is quantifiability of performance. Given the private nature of (motor) imagery, it is inherently difficult to assess whether a subject, when asked to imagine a certain action, is indeed actively engaged in motor imagery. Whereas some studies have simply assumed task compliance (e.g., Ehrsson et al., 2003; Gerardin et al., 2000; Porro et al., 1996), others have included a behavioral component to control for task compliance and aptitude. Sirigu et al. (1996) asked subjects to mentally rehearse a finger opposition sequence to the increasing pace of a metronome (i.e., an explicit motor imagery task). Subjects had to indicate the maximal speed at which they could mentally perform these movements, a measure that could later be compared to the maximal speed of executed finger opposition sequences (Sirigu et al., 1996), making the overall motor imagery performance quantifiable. Similarly, Hanakawa et al. (2003) verified imagery performance during imagined finger tapping by asking subjects to report at unpredictable intervals which finger they were imagining to move while they were engaged in imagery of a predefined movement pattern at a predefined speed. Imagery of more complex actions has been quantified in a similar manner (Bakker et al., 2007; Decety and Jeannerod, 1995; Johnson et al., 2002a; Stevens, 2005).

An influential paradigm that implicitly evokes motor imagery and allows one to quantify performance is the hand-laterality judgment task, in which subjects have to make judgments about rotated images of hands (Parsons, 1987; Sekiyama, 1982). The presence of motor simulations of the left and right hands can be inferred from the behavioral performance. Namely, reaction times (RTs) are not linearly modulated by the rotation of the hand stimulus (as is usually the case during mental rotation paradigms: see Shepard and Cooper, 1982). Rather, RTs closely correspond to the time it would take to execute a similar movement. Biomechanically complex movements (e.g., movements away from the midline of the body) take disproportionally longer than biomechanically easier movements (e.g., movements towards the midline of the body), even if the stimulus rotation is equal (de Lange et al., 2006; Parsons, 1994; Parsons et al., 1998).

There are other examples of implicit motor imagery tasks. One is the grasp judgment task designed by Johnson et al. (2002a), in which a graspable handle is presented in various orientations. Subjects had to judge whether it would be preferable to grasp the handle using an underhand or overhand power grip. This paradigm is similar to the one designed by Frak et al. (2001), in which subjects had to judge the complexity of a grasping movement. In both cases, it is possible to use mental chronometry to quantify the imagery performance of the subject.

\subsection{Motor imagery in healthy subjects}

Using the wide variety of tasks described above, several studies have typically reported a tight correlation between imagined and executed actions along various behavioral dimensions. As already mentioned above, the time it takes to image a certain action is closely correlated with the execution time of the action (Decety and Michel, 1989; Parsons, 
1994; Sirigu et al., 1996; Stevens, 2005). Furthermore, vegetative responses like cardiac and respiratory rhythms covary with the degree of imagined effort (Decety et al., 1991). Motor imagery performance is also influenced by the current state of one's own body, pointing to the embodied nature of this cognitive process. Several studies have found that changing one's body posture affects motor imagery performance (Parsons, 1994; Sirigu and Duhamel, 2001), in an effector-specific manner (de Lange et al., 2006; Shenton et al., 2004).

Several neuroimaging studies have found a host of brain regions that are active during simulated actions [for a metaanalysis, see Grezes and Decety, 2001]. The posterior parietal, premotor and supplementary motor cortex have all been implicated in motor imagery. These regions are also engaged in planning and preparation of movements (Deiber et al., 1996; Rushworth et al., 2003; Toni et al., 2001), suggesting a neural overlap between motor imagery and motor planning and preparation.

In view of the tight link between imagined and executed actions, it has been proposed that the primary motor cortex (M1) may also have a critical role in motor imagery. Several studies have indeed implicated M1 in motor imagery, but this is still an ongoing topic of debate. Neuropsychological studies have found behavioral disturbances during imagined actions in patients with lesions in M1 (Sirigu et al., 1995; Tomasino et al., 2005b). Two transcranial magnetic stimulation (TMS) studies have also found that disruption of M1 selectively interfered with motor imagery performance (Ganis et al., 2000; Tomasino et al., 2005a), although a recent study did not find an involvement of M1 in motor imagery (Sauner et al., 2006). Together, these studies provide some support for a role of M1 in motor imagery, although it should be kept in mind that M1 operates within an interconnected cerebral network, and the effects of a perturbation delivered at one node of a network may influence behavior through changes in other nodes. This consideration applies both to TMS studies (Ruff et al., 2006; Strafella and Paus, 2001) and patient studies (Price and Friston, 2002a; Young et al., 2000). Several electrophysiological studies in humans have also involved motor cortex in motor imagery (Caldara et al., 2004; Carrillo-dela-Pena et al., 2006; McFarland et al., 2000; Pfurtscheller et al., 2006). Neuroimaging methods with higher spatial resolution (like fMRI) have, however, been divided on the issue. While several studies have observed (attenuated) M1 activity during imagery (Dechent et al., 2004; Lacourse et al., 2005; Lotze et al., 1999; Porro et al., 1996; Rodriguez et al., 2004) other studies did not find any M1 activation as a function of imagery, but only M1 activity related to the actual motor response at the end of a trial (de Lange et al., 2005; Richter et al., 2000). Possibly, a host of factors like paradigm choice (e.g., implicit or explicit, simple or complex movements), and subject instructions may contribute to whether or not M1 plays a role during motor imagery (Lotze and Halsband, 2006). Future studies that experimentally manipulate these factors within one design may be of great help to solve this debate.

\subsection{The link between motor imagery and motor execution}

Given the behavioral and neural correlations between imagined actions and actually performed actions, it has been suggested that these processes (at least partly) rely on common mechanisms. More precisely, some authors have suggested that motor imagery relies on the generation of a complete motor plan that is prevented from operating on the body (Grush, 2004; Jeannerod, 1994). However, other authors have suggested that motor imagery relies on processes involved in planning, but not control of movements (Glover, 2004; Johnson et al., 2002b). According to this latter view, there is a dichotomy between the planning system, dealing with action selection before movement onset on the basis of cognitive and visual factors; and the control system, dealing with on-line supervision of movement execution on the basis of motor variables. Therefore, these two frameworks posit that different processes are underlying motor imagery. According to the planning-control framework (Glover, 2004; Johnson et al., 2002b), motor imagery relies on general representations, rather than specific motor representations. An implication of this is that the neural computations that operate on such representations should not be influenced by the current state of one's body. In contrast, according to the simulation/ emulation framework (Grush, 2004; Jeannerod, 1994), motor imagery relies on embodied motor representations. Therefore, motor imagery should depend not only on the desired endstate but also on the current configuration of the limb.

Previous reports have provided evidence supporting either claim. On the one hand, some psychophysical studies failed to find a significant difference in the time required to solve a hand-laterality judgment task by densely hemiplegic and by recovered hemiplegic patients, irrespectively of whether the task involved their paralyzed or their unaffected hand (Johnson, 2000; Johnson et al., 2002b). Furthermore, the patients were as accurate in motor imagery as control subjects that fully recovered from hemiparesis. These results have been taken as evidence that action representations can be independent of one's own body. On the other hand, Nico et al. (2004) showed that the loss of one limb significantly increased the difficulty of performing hand-laterality judgments, notably if the amputated limb was the dominant limb. Similarly, behavioral (Parsons, 1987; Shenton et al., 2004; Sirigu and Duhamel, 2001) and neural (de Lange et al., 2006; Vargas et al., 2004) studies have showed that there is a clear proprioceptive influence on motor imagery performance in healthy subjects, favouring the view that motor imagery relies on the generation of a complete motor plan that is prevented from operating on the body.

\subsection{Motor imagery in pathological conditions}

Motor imagery tasks have been widely used in clinical populations to investigate cognitive aspects of motor dysfunction. For instance, motor imagery impairments have been found in neglect patients (Coslett, 1998), patients with lesions in parietal (Danckert et al., 2002; Sirigu et al., 1996) and motor cortex (Sirigu et al., 1995; Tomasino et al., 2005b), Parkinson's disease (Dominey et al., 1995; Helmich et al., 2007), chronic fatigue syndrome (de Lange et al., 2004), hand dystonia (Fiorio et al., 2006) and patients with peripheral disturbances such as upper limb amputees (Nico et al., 2004), chronic pain patients (Schwoebel et al., 2001) and people with congenital absence of limbs (Funk and Brugger, 2002). There are at least two 
rationales for using motor imagery paradigms in patient populations. First, one can test whether a given impairment affects motor processing beyond simple execution [see for instance, Schwoebel et al., 2001]. Second, for motor disorders that do not impair motor imagery performance, one can probe movement-related processes using a task that the patient can perform, while allowing for objective measures of patients' performance and strategies. This is a necessary requirement if one wants to attribute behavioral performance and/or cerebral activity to pathological mechanisms (Price and Friston, $2002 b)$, rather than unspecific factors related to impaired performance.

Recently, the study of motor cognitive impairments has been extended to psychopathological conditions. For instance, some authors have tested the hypothesis that the motor passivity of some schizophrenic patients might be linked to altered generation of forward models in the parietal cortex (Danckert et al., 2004; Maruff et al., 2003). CP is another psychopathological condition for which motor imagery is a viable tool to gain insight in the underlying pathological mechanisms. CP is a syndrome characterized by a loss of motor function without apparent 'organic' cause. There are competing theories about the functional mechanisms behind this syndrome. Some studies suggest the disorder is characterized by inhibition of movement plans (Halligan et al., 2000; Marshall et al., 1997). Other studies claim that the disorder is associated with heightened selfmonitoring during actions (Roelofs et al., 2006; Vuilleumier et al., 2001). Recently, we have used an implicit and explicit motor imagery paradigm in order to test the predictions of these competing theories.

\section{5. $\mathrm{CP}$}

CP is a mental disorder characterized by loss of voluntary motor functioning. Although the symptoms may suggest a neuropathological condition, they cannot be adequately explained by known neurological or other organic disorders (American Psychiatric Association, 1994). Moreover, there is an exacerbation of symptoms at times of psychological stress, which suggests that psychological mechanisms play a role. Conversion disorder and related disorders are common in clinical practice. About one third of new neurological outpatients exhibit medically unexplained symptoms (Carson et al., 2000; Stone et al., 2005). Despite its high prevalence among neurological outpatients, little is known about the neurobiological basis of this motor dysfunction, and its functional neuro-anatomy is controversial. Several studies have investigated the functional neuro-anatomy of $\mathrm{CP}$ by recording brain activity during attempted movement of the paralyzed limb (Burgmer et al., 2006; Marshall et al., 1997; Spence et al., 2000) but different studies obtained conflicting results. One of the reasons for the inconsistency may be that patients were asked to carry out a task ("move/try to move your affected limb") that they could not appropriately perform due to their condition. Accordingly, it is conceivable that these results reveal cerebral effects related to the cognitive consequences of a failed movement (like altered effort, motivation, or error processing), rather than impaired formation of action representations. Motor imagery can overcome some of these interpretational issues, since it does not rely on actual motor execution but still taps into the motor system. Previous behavioral studies have used motor imagery tasks to reveal impairments in motoric simulations of the affected limb in patients with CP (Maruff and Velakoulis, 2000; Roelofs et al., 2001).

We recently tested the hypothesis that CP can be linked to heightened self-monitoring. Heightened self-monitoring is associated with increased behavioral inhibition in patients with anxiety disorders (Gehring et al., 2000; Hajcak and Simons, 2002; Ursu et al., 2003). In view of the stress-induced immobility observed in CP, we hypothesized that heightened self-monitoring may play a functional role also in this disorder (Roelofs et al., 2006). We found that implicit motor imagery of the affected hand leads to stronger responses in the superior temporal and ventromedial prefrontal cortex (de Lange et al., 2007) compared to the unaffected hand. These regions have been associated with self-reflexive processing (Goldberg et al., 2006), as well as observation and awareness of actions (Castelli et al., 2000; Frith et al., 2000), substantiating the link between $\mathrm{CP}$ and heightened self-monitoring during actions with the affected arm.

In the current study, we have tested a prediction of this interpretation. Namely, if the altered pattern of activity of those regions is related to increased self-monitoring for imagined actions of the affected hand, then inducing self-monitoring of actions of the unaffected limb (by means of explicitly cued motor imagery) should abolish the activation differences observed during implicit motor imagery.

\section{Materials and methods}

\subsection{Participants}

We studied seven patients (mean age of 31.6 years, range $18-48, \mathrm{SD}=10.8$ ) diagnosed with conversion disorder according to the DSM-IV criteria (American Psychiatric Association, 1994) and showing a full or partial paralysis lateralized to one arm as a major symptom. For a full description of inclusion criteria and diagnosis procedure, see (de Lange et al., 2007). Four patients showed conversion paresis to the right arm and three patients showed conversion paresis to the left arm. Lateralization of the paresis was examined by measuring maximal contraction force. Isometric force measurements of maximum voluntary contractions (MVCs) of the left and right hand were obtained with a Biometrics hand dynamometer (Almere, Netherlands). Force measures confirmed that the maximal force that could be exerted with the affected arm was considerably lower than with the unaffected hand in all patients $\left(t_{(6)}=5.52, p=.001\right)$, although the loss of motor function was not total in the affected arm. One patient used antidepressant medication (Sertraline, $50 \mathrm{mg} /$ day). None of the patients used anti-convulsants, benzo-diazepines, or other substances that are known to have an effect on cerebral blood flow. Table 1 shows demographic information of all the participants. The study was approved by the local medical ethical committee and all patients gave their informed consent before participation. 
Table 1 - Demographical characteristics of the participants

\begin{tabular}{|c|c|c|c|c|c|c|c|c|c|c|}
\hline Patient & Age & Gender & $\begin{array}{l}\text { Affected } \\
\text { hand }\end{array}$ & $\begin{array}{l}\text { Dominant } \\
\text { hand }\end{array}$ & $\begin{array}{l}\text { Duration of } \\
\text { complaints }^{\mathrm{a}}\end{array}$ & $\begin{array}{c}\text { MVC }^{\mathrm{b}} \\
\text { affected }\end{array}$ & $\begin{array}{c}\text { MVC }^{\mathrm{b}} \\
\text { unaffected }\end{array}$ & $\begin{array}{l}\text { History of } \\
\text { traumatic } \\
\text { events }\end{array}$ & $\begin{array}{l}\text { Events preceding } \\
\text { symptom onset }\end{array}$ & $\begin{array}{c}\text { Axis-I } \\
\text { comorbidity } \\
\text { (SCID-I) }\end{array}$ \\
\hline 1 & 48 & Female & Right & Right & 36 & 100.8 & 139.4 & $\begin{array}{l}\text { Emotional and } \\
\text { sexual abuse }\end{array}$ & Family conflict & $\begin{array}{l}\text { Depressive disorder } \\
\text { in remission }\end{array}$ \\
\hline 2 & 34 & Male & Left & Right & 35 & 157.2 & 219.4 & - & $\begin{array}{l}\text { Suicide attempt } \\
\text { by sibling }\end{array}$ & - \\
\hline 3 & 43 & Female & Right & Right & 3 & 8.9 & 106.8 & $\begin{array}{l}\text { Sexual and } \\
\text { physical abuse }\end{array}$ & Family conflict & - \\
\hline 4 & 23 & Female & Right & Right & 41 & 59.3 & 139.4 & - & Car accident & - \\
\hline 5 & 27 & Male & Left & Left & 26 & 172.0 & 261.0 & - & Work accident & - \\
\hline 6 & 28 & Female & Right & Right & 19 & 86.0 & 127.5 & - & School exam & - \\
\hline 7 & 18 & Female & Left & Right & 3 & 4.4 & 154.2 & $\begin{array}{l}\text { Emotional } \\
\text { abuse; left } \\
\text { arm fracture }\end{array}$ & $\begin{array}{l}\text { Panic attack, } \\
\text { change of living } \\
\text { situation }\end{array}$ & $\begin{array}{l}\text { Anxiety disorder } \\
\text { not otherwise } \\
\text { specified }\end{array}$ \\
\hline
\end{tabular}

a In months.

b MVC in Newtons, measured with a hand dynamometer.

\subsection{Task}

All patients engaged in an implicit motor imagery experiment and an explicit motor imagery experiment. For both experimental sessions, we used a well-known motor imagery task, in which the participants have to judge the laterality of the visually presented rotated hand stimulus (Parsons, 1987). We used line drawings of left and right hands, in different orientations varying from $0^{\circ}$ to $180^{\circ}$ in $45^{\circ}$ steps (both clockwise and counter-clockwise), and in palmar and dorsal orientations. The stimuli were serially presented to the patients in a random order. During implicit motor imagery, patients were instructed to judge as fast and as accurately as possible whether the stimulus was a left or a right hand. During explicit motor imagery, patients were instructed to imagine that the hand on screen was their own hand, and imagine moving their own hand from the position on screen to the upright position, and then finally to indicate whether the stimulus constituted a left or a right hand. As such, this task is identical to implicit motor imagery, except for the fact that the subjects are explicitly instructed to vividly imagine their own hand rotating from the current position into the presented target position. After the patients provided their response, the stimulus was replaced with a fixation cross, which stayed on until the start of the next trial (inter-trial interval: $1.5-2.5 \mathrm{sec}$ ).

In order to avoid carry-over effects of explicit motor imagery to implicit motor imagery, the implicit motor imagery session always preceded the explicit motor imagery session.

Each fMRI scanning session consisted of 160 trials of motor imagery. After a series of 10 motor imagery trials, a rest period of $10 \mathrm{sec}$ was introduced to sample baseline activity. During this rest period, patients were instructed to look at the fixation cross. The explicit imagery experiment was preceded by a short imagery training (outside the MR-scanner), in which the patients were asked to imagine several motor actions. Also, each series of motor imagery trials during the explicit motor imagery experiment (inside the MR-scanner) was preceded by a short imagery induction phase, in which subjects were asked to imagine a certain action (e.g., "imagine touching your nose with your right arm") to motivate the subjects to keep engaged in explicit motor imagery.

Patients responded by pressing one of two buttons attached to their left or right big toe. The patients' left and right feet were firmly attached to a button box, and RTs and error rates were measured for subsequent behavioral analysis. The stimuli were presented using Presentation software (Neurobehavioral systems, Albany, USA), and they were projected onto a screen at the back of the scanner and seen through a mirror above the patients' heads.

\subsection{Behavioral analysis}

Mean RTs were calculated for each level of the two experimental factors (hand, rotation) and each experimental condition (implicit imagery, explicit imagery). A three-way $(2 \times 2 \times 5)$ repeated-measures analysis of variance (ANOVA) was carried out to examine the effects of experimental set (implicit, explicit), hand (affected, unaffected) and rotation ( $0^{\circ}$ to $180^{\circ}$ in $45^{\circ}$ steps) on RT. Additionally, we investigated whether RTs were influenced by the biomechanical complexity of the imagined movements. To this end, we carried out a three-way $(2 \times 2 \times 5)$ repeated-measures ANOVA with the factors experimental set (implicit, explicit), hand (left, right) and direction of rotation (clockwise, counter-clockwise). Clockwise orientations are biomechanically easier for left hands, while counter-clockwise orientations are biomechanically easier for right hands (Parsons, 1994). Differences in error rate between the affected and the unaffected hand for each experiment were investigated using a paired-samples T-test.

\subsection{MRI acquisition and analysis}

Functional images were acquired on a Siemens (Erlangen, Germany) 1.5 T MRI system equipped with echo planar imaging (EPI) capabilities using the standard head coil for radio frequency transmission and signal reception. Functional images were acquired using a gradient EPI-sequence (TE/ $\mathrm{TR}=40 / 2540 \mathrm{msec} ; 32$ axial slices, voxel size $=3.5 \mathrm{~mm}$; FOV $=$ $224 \mathrm{~mm}$ ). On average, the duration of the implicit and explicit 
motor imagery experiments was $23 \mathrm{~min}$ (547 scans) and $35 \mathrm{~min}$ (818 scans), respectively. High-resolution anatomical images were acquired using a MP-RAGE sequence (TE/ $\mathrm{TR}=3.93 / 2250 \mathrm{msec}$; voxel size $=1.0 \mathrm{~mm}, 176$ sagittal slices; $\mathrm{FOV}=256 \mathrm{~mm}$ ). Preprocessing of the functional data and calculation of the contrast images for statistical analysis was done with Statistical Parametric Mapping (SPM5; www.fil. ion.ucl.ac.uk/spm). First, functional images were realigned, slice-time corrected, normalized to a common stereotactic space (MNI: Montreal Neurological Institute, Canada) and smoothed with a $10 \mathrm{~mm}$ FWHM Gaussian kernel. By jittering trial onsets with respect to image acquisition and randomizing stimulus rotations, our experimental design allowed for an event-related analysis of the fMRI time series. For each experimental session of each patient, we modeled the overall activity evoked by motor imagery (2 levels: affected vs. unaffected) as a train of delta functions, of which the onset corresponded to the onset of the stimulus presentation, and the duration corresponded to the average RT of the session of that subject. Each regressor was then convolved by the haemodynamic response function. Moreover, we modeled the increase in activity with increasing rotation during motor imagery ( 5 levels: from $0^{\circ}$ to $180^{\circ}$ in $45^{\circ}$ steps), as a linear parametric modulation that was orthogonal to the regressor describing overall motor imagery-related activity. The laterality of the affected hand was pooled across subjects. In other words, activation differences between the affected and unaffected hand considered both patients who were affected on the left side and patients who were affected on the right side. Incorrect responses, and induction trials (for explicit motor imagery) were separately included in the model as events of no interest. To remove any artifactual signal changes due to head motion, we included six parameters describing the headmovements (three translations, three rotations) as confounds in the model. Linear contrasts pertaining to the main effects of the factorial design constituted the data for the second-stage analysis, which treated participants as a random factor. In this second-stage analysis, we tested for overlap and differences between implicit and explicit motor imagery for the following contrasts: (1) increases in activity with increasing motor imagery complexity (as parameterized by the regressors describing the rotation-related increase) versus baseline; and (2) differences in activity between motor imagery of the affected and the unaffected hand. For each contrast, we tested the significance of the comparison for implicit and explicit motor imagery tasks separately, using one-sample T-tests. We also tested for significant differences of the contrasts between implicit and explicit motor imagery, using paired-samples T-tests. In view of the low number of subjects, we used a region of interest approach on the basis of anatomical information derived from previous studies. The search space for the contrast that tested for increasing activity with increasing rotation was constrained on the basis of previously published coordinates of the left and right intraparietal sulcus and dorsal premotor cortex (de Lange et al., 2005), while we tested for activity differences between the affected and the unaffected hand in a search space spanning the medial prefrontal and left and right superior temporal cortex, again based on previously published coordinates (de Lange et al., 2007). For each comparison, we first set a voxel-wise threshold of $p<.001$. Then, we defined spheres with a radius of $10 \mathrm{~mm}$ around the regions of interest, and corrected our results for multiple comparisons within the search space using the false discovery rate (Genovese et al., 2002), with a threshold of $p<.05$ corrected.

Anatomical details of activated clusters were obtained by superimposing the SPMs on the structural images of the patients.

\section{Results}

\subsection{Behavioral results}

RTs of the participants for each task are shown in Fig. 1. Subjects were overall slower for explicit motor imagery than for implicit motor imagery (main effect of task: $F_{(1,5)}=16.7$; $p=.009$ ). RTs were modulated by stimulus rotation (main effect of rotation: $\left.F_{(4,20)}=18.6 ; p<.001\right)$. A trend analysis showed that RTs were linearly modulated by stimulus rotation $\left(F_{(1,5)}=42.3 ; p<.001\right)$. Although RTs appeared slightly longer for the affected hand than for the unaffected hand in both tasks, this effect was not statistically significant (main effect of hand: $\left.F_{(1,5)}=1.61 ; p=.26\right)$. There were no significant twoor three-way interactions between task, hand, and rotation (all $p>.10$ ). To investigate whether the RT profiles followed the biomechanical properties of the arm during the implicit and explicit motor imagery tasks, we carried out a three-way
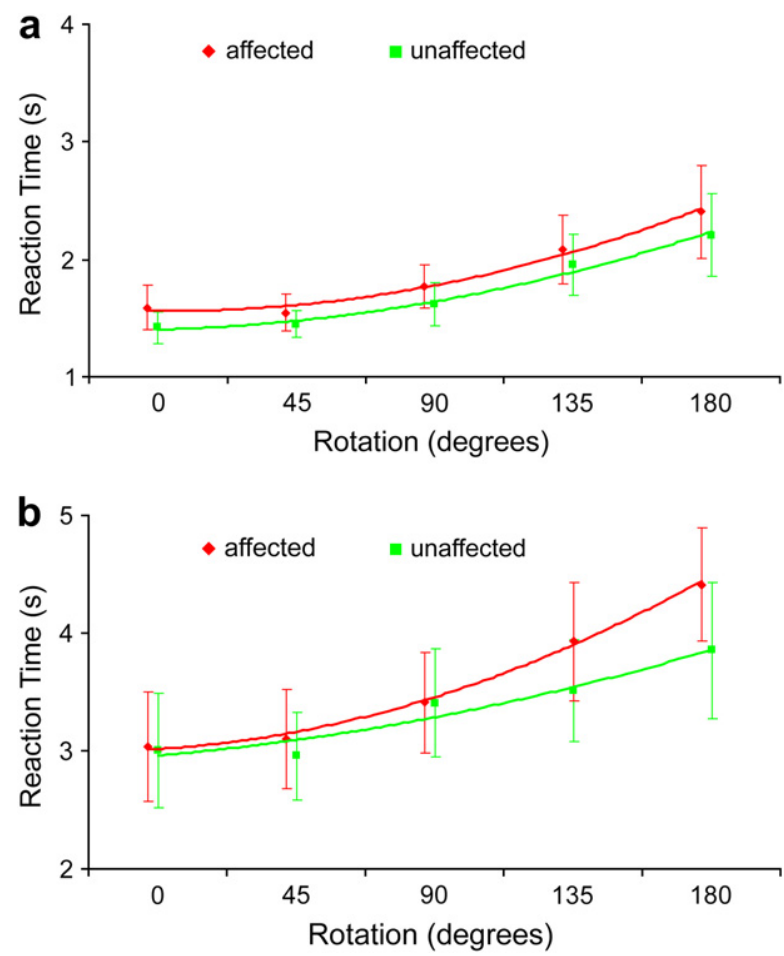

Fig. 1 - Behavioral data. (a) RTs (mean \pm SEM) for implicitly induced motor imagery. RTs for the affected hand are plotted in red, and for the unaffected hand in green. (b) RTs (mean \pm SEM) for explicitly motor imagery. RTs for the affected hand are plotted in red, and for the unaffected hand in green. 
$(2 \times 2 \times 2)$ repeated-measures ANOVA with experimental set (implicit, explicit), hand (left, right) and direction of rotation (clockwise, counter-clockwise) as experimental factors. If the RTs follow the biophysical properties of the arm, trials in CW orientations should be faster for left hands, whereas trials in CCW orientations should be faster for right hands (Parsons, 1994; Parsons et al., 1998). Indeed, subjects were slower for left hands in counter-clockwise orientations than for left hands in clockwise orientations (mean difference $=152 \mathrm{msec}$ ), while subjects were faster for right hands in counter-clockwise orientations than for right hands in clockwise orientations (mean difference $=-198 \mathrm{msec}$ ) during both implicit and explicit motor imagery. This resulted in a hand $\times$ orientation interaction that tended towards significance $\left(F_{(1,6)}=4.17\right.$; $p=.087)$. Crucially, the interaction was not different for the implicit and explicit motor imagery task (task $\times$ hand $\times$ orientation: $\left.F_{(1,6)}=.33 ; p=.59\right)$. This suggests that subjects imagined moving their own hand rotating into the displayed hand during both conditions.

All patients performed with low error rates during implicit motor imagery (affected hand: 6.3\%; unaffected hand: $6.4 \%$ ) and during explicit motor imagery (affected hand: $3.8 \%$; unaffected hand: $8.0 \%$ ). There was no difference in error rate between implicit and explicit motor imagery $\left(F_{(1,6)}=.13\right.$; $p=.73)$, or between affected and unaffected hands $\left(F_{(1,6)}=\right.$ 3.23; $p=.12$ ). There was also no interaction between these factors $\left(F_{(1,6)}=1.80 ; p=.23\right)$.

\subsection{Cerebral effects - common increase in parietal and premotor activity during implicit and explicit motor imagery}

There were significant increases in dorsal parietal and premotor cortex with increasing rotation, both during implicit and explicit motor imagery. Fig. 2 illustrates the anatomical location of these regions showing a significant increase in activity with increasing mental rotation. These increases were remarkably similar during both tasks, and similar for the affected and unaffected hand. These results are well in line with previous studies showing an involvement of parietal and premotor cortex in imagined hand actions (de Lange et al., 2005, 2006; Johnson et al., 2002a). There were no regions showing a significant difference in their rotation-related activity increase between implicit and explicit imagery, or between the affected and the unaffected hand.

\subsection{Cerebral effects -activity differences between the affected and unaffected hand during implicit and explicit motor imagery}

As described previously (de Lange et al., 2007), superior and medial portions of the frontal cortex, the gyrus rectus (Chiavaras and Petrides, 2000) and superior temporal cortex showed greater cerebral activity for the affected hand than the unaffected hand during implicit motor imagery (see Fig. 3 and Table 3). The activity patterns show that these effects relate to reduced responses during implicit motor imagery of the unaffected hand with respect to the baseline (Fig. 3b).

These activation differences between the affected and the unaffected hand were not present during explicit motor imagery. A direct statistical comparison between implicit and explicit motor imagery confirmed that the activity difference between the affected and the unaffected hand in the medial prefrontal cortex was specific to implicit motor imagery (see Table 3).

There were no clusters showing greater overall activity during motor imagery of the unaffected hand compared to the affected hand during implicit or explicit imagery.

\section{Discussion}

In this paper, we have reviewed different approaches and rationales for using motor imagery to study motor cognition in humans, as well as its application to neurological and neuropsychiatric disorders. We have illustrated how the application of motor imagery in conjunction with neuroimaging methods has been used to shed light on an ill-understood neuropsychiatric condition, CP. This approach has generated a specific prediction on the behavioral and cerebral effects of implicitly or explicitly inducing action simulations in these patients, and we have reported an empirical test of this prediction.

\subsection{Motor simulation and action monitoring in $\mathrm{CP}$}

Behavioral results supported the notion that CP patients engaged in a motor simulation of their own hand, during both implicitly induced and explicitly evoked motor imagery. Moreover, both tasks evoked remarkably similar patterns of activity within the motor system of the CP patients. Cerebral activity in dorsal parietal and premotor cortex (Fig. 2) increased linearly with increasing amount of mental rotation, during both implicit and explicit motor imagery, for the affected as well as for the unaffected limb. This same parieto-premotor network has also been isolated in earlier studies using similar imagery paradigms (de Lange et al., 2005; Ecker et al., 2006; Johnson et al., 2002a; Kawamichi et al., 2007; Lamm et al., 2007; Richter et al., 2000), as well as during the selection and preparation of actual hand movements (Rushworth et al., 2003; Thoenissen et al., 2002; Toni et al., 1999). The matched contribution of these motor regions to implicit and explicit imagery suggests that both tasks evoked motor simulation of hand actions to a similar degree, and that, as far as the motor system is concerned, explicit and implicit motor imagery were indistinguishable. In other words, since there were no differences in these motor structures between imagined actions of the affected and the unaffected hand, the paralysis that characterizes conversion patients is unlikely to originate from altered motor processing.

Beside these commonalities, there were also important differences between implicit and explicit motor imagery, both at the behavioral and at the cerebral level. Behaviorally, explicit imagery was characterized by longer RTs than implicit motor imagery, mimicking results of an earlier study (Roelofs et al., 2001) and likely related to the additional task demands.

Crucially, the cerebral data showed differences between motor imagery of the affected and the unaffected hand that were dependent on whether the task was implicitly induced or explicitly evoked. While implicit imagery was characterized by a larger activation in the ventromedial prefrontal and superior temporal cortex during imagined actions of the affected 
a

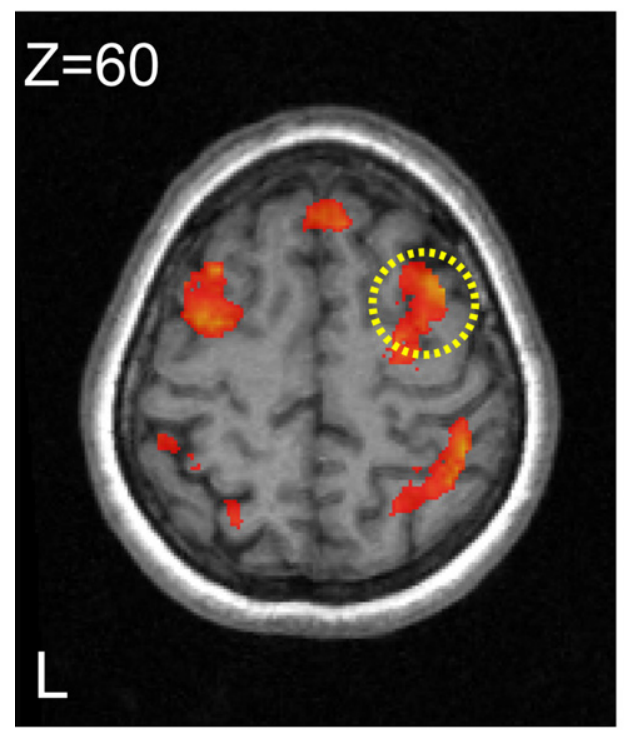

C

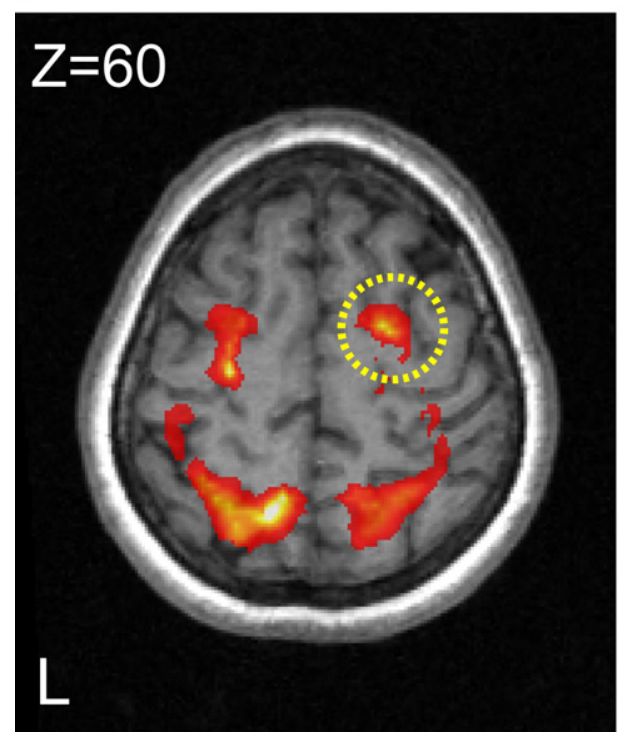

b

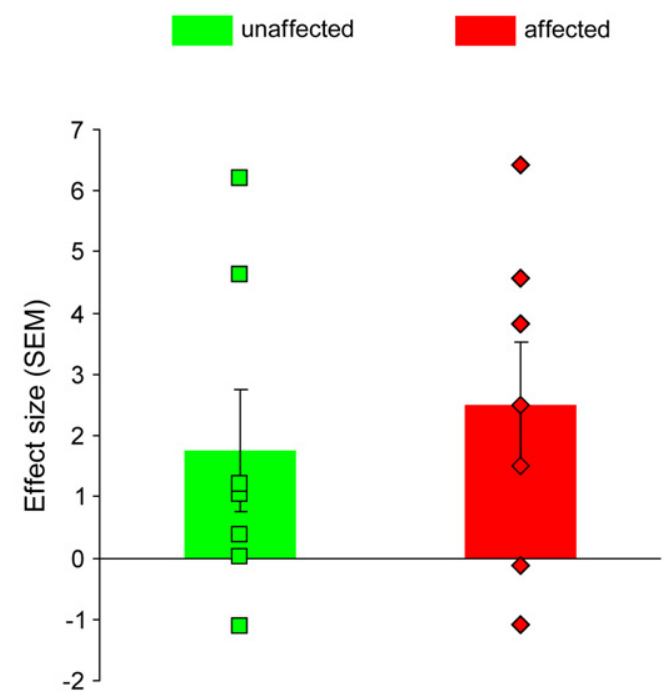

d

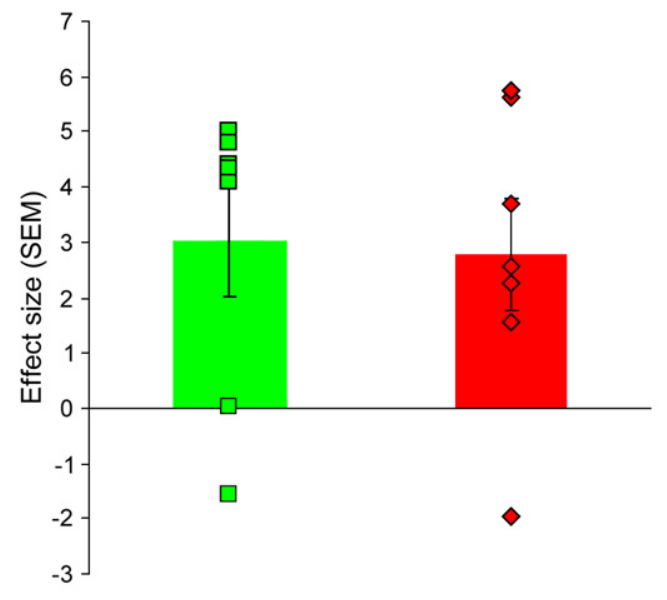

Fig. 2 - Common rotation-related increases in cerebral activity. Left row: Anatomical localization of regions showing a significant linear increase in activity with increasing stimulus rotation for both hands during implicitly induced motor imagery (a) and explicitly evoked motor imagery (c). The statistical maps are thresholded at $T>3.0$, for visualization purposes. Dotted circles denote the regions of which the effect sizes are plotted. Right row: Effect size $( \pm \mathrm{SEM})$ of the parametric effect in the right dorsal precentral sulcus during implicitly induced motor imagery (b) and explicitly evoked motor imagery (d). In view of the low number of subjects, we have plotted the individual responses on top of the average effect size. Dots on the histograms denote individual data points. As can be seen from the figure, linear increases in cerebral activity with rotation were positive in $5 / 7$ or more subjects for implicit and explicit motor imagery of the affected and unaffected hand. Exact stereotactic coordinates are given in Table 2.

hand compared to the unaffected hand, this between-hands difference was absent during explicit motor imagery. These differences can be understood in terms of the different load that implicit and explicit imagery impose on self-monitoring. The ventromedial prefrontal cortex is part of the "intrinsic" or "default" network (Raichle and Mintun, 2006), showing physiological decreases of metabolic activity during performance of sensorimotor and cognitive tasks (Gusnard et al., 2001). When healthy subjects are engaged in a demanding task, metabolic activity in the prefrontal cortex is decreased as compared to when subjects are engaged in self-reflexive processing (Goldberg et al., 2006). The disappearance of this activity reduction during implicit motor imagery of the affected hand is in line with the notion that, in CP patients, 
a

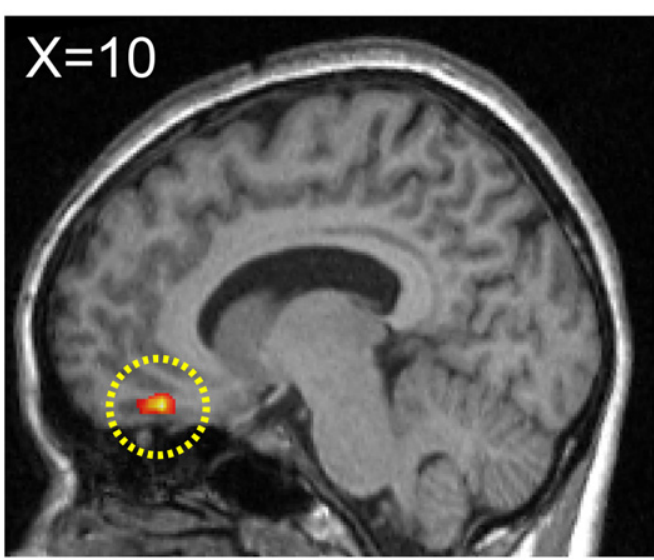

C

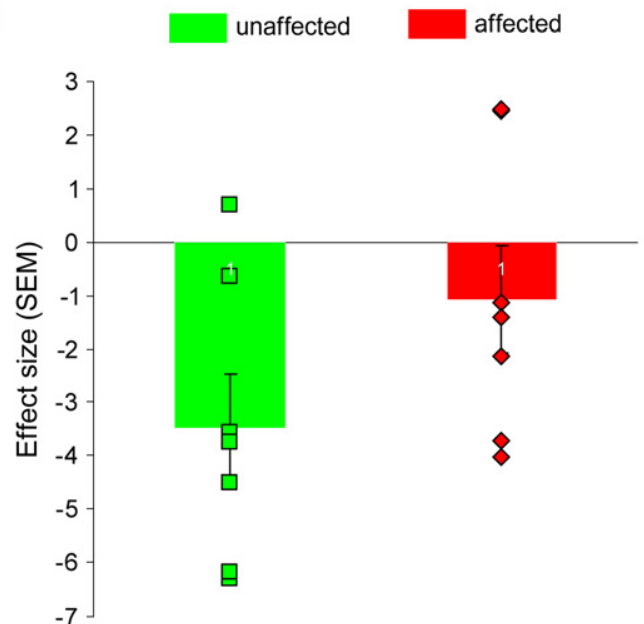

b

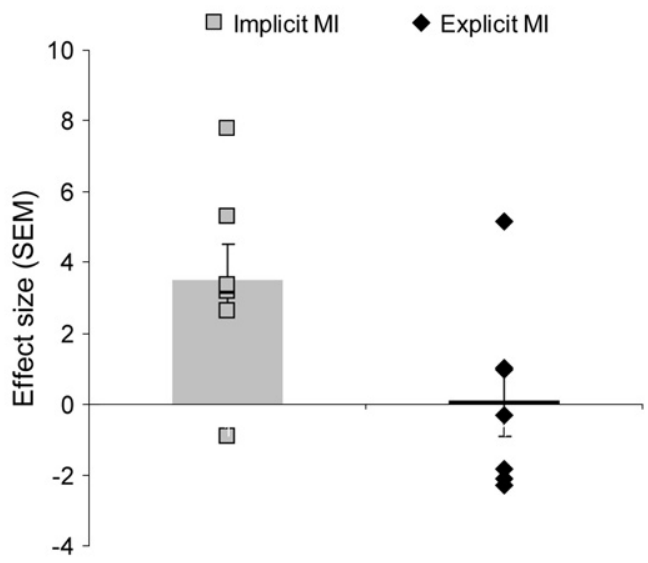

d

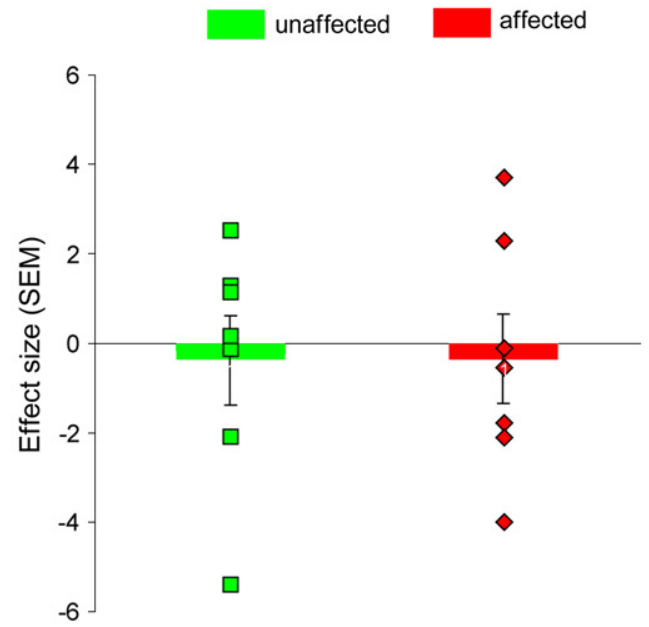

Fig. 3 - Cerebral differences between motor imagery of the affected and the unaffected hand between implicit and explicit motor imagery. (a) Anatomical localization of a ventromedial prefrontal cluster, showing overall (i.e., not rotation-related) decreased de-activation for the affected hand during implicit motor imagery, but no activation differences between hands during explicit motor imagery. (b) Effect size $( \pm$ SEM) of activation difference between the affected and unaffected hand during implicit motor imagery (grey squares) and during explicit motor imagery (black diamonds). Dots on the histograms denote individual data points. As can be seen from the figure, there was a positive difference between activity for the affected and the unaffected hand in the ventromedial prefrontal cortex in 6/7 subjects during implicit motor imagery, while there was no consistent difference during explicit motor imagery. (c) Effect size of activation differences with respect to baseline $( \pm$ SEM) during implicit motor imagery, for the unaffected and the affected arm. (d) Effect size of activation differences w.r.t. baseline $( \pm \mathrm{SEM})$ during explicit motor imagery, for the unaffected and the affected arm. Dots on the histograms denote individual data points. Exact stereotactic coordinates are given in Table 3. Other conventions as in Fig. 2.

\section{Table 2 - Cerebral data - areas showing increasing activity with rotation}

\begin{tabular}{|c|c|c|c|c|c|c|}
\hline \multirow[t]{2}{*}{ Task } & \multirow[t]{2}{*}{ Region } & \multirow[t]{2}{*}{$\mathrm{T}$} & \multirow[t]{2}{*}{ Corrected $p$-value } & \multicolumn{3}{|c|}{ Stereotactic coordinates } \\
\hline & & & & $x$ & $\mathrm{y}$ & $z$ \\
\hline \multirow[t]{3}{*}{ Implicit MI } & Intraparietal sulcus & 5.4 & .033 & 28 & -62 & 52 \\
\hline & Dorsal precentral sulcus & 9.7 & .006 & -28 & -4 & 66 \\
\hline & & 9.8 & .006 & 28 & 8 & 64 \\
\hline \multirow[t]{4}{*}{ Explicit MI } & Intraparietal sulcus & 8.4 & .01 & -28 & -56 & 64 \\
\hline & & 10.0 & .003 & 26 & -60 & 58 \\
\hline & Dorsal precentral sulcus & 8.1 & .021 & -20 & -4 & 60 \\
\hline & & 6.2 & $.054^{\mathrm{a}}$ & 22 & -2 & 58 \\
\hline
\end{tabular}

All reported coordinates are in MNI space. Stereotactic coordinates denote the peak of the voxels surviving correction for multiple comparisons. a This cluster did not survive correction for multiple comparisons. 
Table 3 - Cerebral data - areas showing greater activity for motor imagery of the affected than the unaffected hand

\begin{tabular}{|c|c|c|c|c|c|c|}
\hline \multirow[t]{2}{*}{ Task } & \multirow[t]{2}{*}{ Region } & \multirow[t]{2}{*}{$\mathrm{T}$} & \multirow[t]{2}{*}{ Corrected $p$-value } & \multicolumn{3}{|c|}{ Stereotactic coordinates } \\
\hline & & & & $x$ & $y$ & $\mathrm{z}$ \\
\hline \multirow[t]{4}{*}{ Implicit MI } & Gyrus rectus & 13.5 & .001 & 10 & 38 & -22 \\
\hline & Medial frontal gyrus & 9.5 & .005 & -12 & 60 & 32 \\
\hline & Superior frontal gyrus & 5.9 & .026 & -32 & 48 & 36 \\
\hline & Superior temporal cortex & 6.1 & $.066^{\mathrm{a}}$ & -58 & -14 & 8 \\
\hline \multirow[t]{3}{*}{ Implicit > explicit MI } & Gyrus rectus & 5.9 & .028 & 12 & 36 & -20 \\
\hline & Medial frontal gyrus & 7.8 & .020 & -10 & 56 & 34 \\
\hline & Superior frontal gyrus & 7.8 & .022 & -32 & 42 & 30 \\
\hline
\end{tabular}

All reported coordinates are in MNI space. Stereotactic coordinates denote the peak of the voxels surviving correction for multiple comparisons. a This cluster did not survive correction for multiple comparisons.

simulating movements of the affected hand is associated with increased self-monitoring processes (Roelofs et al., 2006; Vuilleumier, 2005). Increased self-monitoring may play a functional role in this disorder. In correspondence with this, heightened self-monitoring has been observed in patients with other stress-related disorders (Gehring et al., 2000; Hajcak and Simons, 2002; Ursu et al., 2003). Explicitly instructing the patients to imagine moving their hand may have driven them towards an increased self-monitoring of their own actions. Accordingly, we found that, during explicit motor imagery, the reduction of activity in the medial prefrontal cortex disappeared for both the affected and unaffected hand, abolishing the between-hands difference observed during implicit motor imagery. This finding suggests that implicit and explicit motor imagery, though identical at the level of motoric simulations, have a differential load on selfmonitoring of actions and in particular on medial prefrontal responses. This notion fits well with the therapeutical observation that overt training of motor skills (cf. explicit motor imagery), which is common practice in revalidation, does not always improve symptoms in CP. For this reason therapeutical programs often make use of indirect techniques (cf. implicit motor imagery) like hypnosis in order to elicit movements (Moene and Roelofs, 2007; Moene et al., 1998).

\section{Conclusions}

There is abundant evidence from psychophysical and neuroimaging studies, as well as from patient studies in neurological and psychiatric populations that motor imagery can provide a window into the mechanisms and alterations of motor cognition (Jeannerod, 2006). Although the behavioral and neural signature of motor imagery in the healthy brain, as well as its possible disturbances, has been investigated in detail, this has not yet led to a wide use of motor imagery as a diagnostic tool. The reason for this may in part be due to the multitude of variables that can influence cognitive processes and subjects' strategies, like the motor imagery paradigm used, the tools used to investigate behavioral or neural performance, and psychological factors like motivation. Here we have illustrated the influence of one variable, selfmonitoring, on behavioral and neural performance in a group of patients with $\mathrm{CP}$, by comparing implicitly induced and explicitly evoked motor imagery. We have shown that imagery tasks induce not only robust motor-related cerebral and behavioral responses, but also self-monitoring activities that are sensitive to task instructions. These findings might be relevant for improving the reliability of current applications of motor imagery as diagnostic or therapeutic tools.

\section{Acknowledgments}

FdL and IT were supported by Dutch Science Foundation (NWO: VIDI grant no. 452-03-339). KR was supported by Dutch Science Foundation (NWO VENI grant no. 451-02-115). This study was supported by the Dutch Brain Foundation (Hersenstichting Nederland, grant number 12F04(2).19) awarded to KR and FdL. The authors would like to thank Marije van Beilen for her generous assistance in recruiting patients, and Paul Gaalman for expert assistance during scanning.

\section{R E F E R E N C E S}

American Psychiatric Association. Diagnostic and Statistical Manual of Mental Disorders. 4th ed. Washington DC: American Psychiatric Press, 1994.

Bakker M, de Lange FP, Stevens JA, Toni I, and Bloem BR. Motor imagery of gait: a quantitative approach. Experimental Brain Research, 179: 497-504, 2007.

Burgmer M, Konrad C, Jansen A, Kugel H, Sommer J, Heindel W, Ringelstein EB, Heuft G, and Knecht S. Abnormal brain activation during movement observation in patients with conversion paralysis. NeuroImage, 29: 1336-1343, 2006.

Caldara R, Deiber MP, Andrey C, Michel CM, Thut G, and Hauert CA. Actual and mental motor preparation and execution: a spatiotemporal ERP study. Experimental Brain Research, 159: 389-399, 2004.

Carrillo-de-la-Pena MT, Lastra-Barreira C, and Galdo-Alvarez S. Limb (hand vs. foot) and response conflict have similar effects on event-related potentials (ERPs) recorded during motor imagery and overt execution. European Journal of Neuroscience, 24: 635-643, 2006.

Carson AJ, Ringbauer B, Stone J, McKenzie L, Warlow C, and Sharpe M. Do medically unexplained symptoms matter? A prospective cohort study of 300 new referrals to neurology outpatient clinics. Journal of Neurology Neurosurgery and Psychiatry, 68: 207-210, 2000. 
Castelli F, Happe F, Frith U, and Frith C. Movement and mind: a functional imaging study of perception and interpretation of complex intentional movement patterns. NeuroImage, 12: 314-325, 2000.

Chiavaras MM and Petrides M. Orbitofrontal sulci of the human and macaque monkey brain. Journal of Comparative Neurology, 422: 35-54, 2000.

Coslett HB. Evidence for a disturbance of the body schema in neglect. Brain and Cognition, 37: 527-544, 1998.

Danckert J, Ferber S, Doherty T, Steinmetz H, Nicolle D, and Goodale MA. Selective, non-lateralized impairment of motor imagery following right parietal damage. Neurocase, 8: 194-204, 2002.

Danckert J, Saoud M, and Maruff P. Attention, motor control and motor imagery in schizophrenia: implications for the role of the parietal cortex. Schizophrenia Research, 70: 241-261, 2004.

Decety J and Jeannerod M. Mentally simulated movements in virtual reality: does Fitts's law hold in motor imagery? Behavioural Brain Research, 72: 127-134, 1995.

Decety J, Jeannerod M, Germain M, and Pastene J. Vegetative response during imagined movement is proportional to mental effort. Behavioural Brain Research, 42: 1-5, 1991.

Decety J and Michel F. Comparative analysis of actual and mental movement times in two graphic tasks. Brain and Cognition, 11: 87-97, 1989 .

Dechent $\mathrm{P}$, Merboldt KD, and Frahm J. Is the human primary motor cortex involved in motor imagery? Cognitive Brain Research, 19: 138-144, 2004.

Deiber MP, Ibanez V, Sadato N, and Hallett M. Cerebral structures participating in motor preparation in humans: a positron emission tomography study. Journal of Neurophysiology, 75: 233-247, 1996.

Dijkerman HC, Letswaart M, Johnston M, and Macwalter RS. Does motor imagery training improve hand function in chronic stroke patients? A pilot study. Clinical Rehabilitation, 18: 538-549, 2004.

Dominey P, Decety J, Broussolle E, Chazot G, and Jeannerod M. Motor imagery of a lateralized sequential task is asymmetrically slowed in hemi-Parkinson's patients. Neuropsychologia, 33: 727-741, 1995.

Ecker C, Brammer MJ, David AS, and Williams SC. Time-resolved fMRI of mental rotation revisited-dissociating visual perception from mental rotation in female subjects. NeuroImage, 32: 432-444, 2006.

Ehrsson HH, Geyer S, and Naito E. Imagery of voluntary movement of fingers, toes, and tongue activates corresponding body-part-specific motor representations. Journal of Neurophysiology, 90: 3304-3316, 2003.

Fiorio M, Tinazzi M, and Aglioti SM. Selective impairment of hand mental rotation in patients with focal hand dystonia. Brain, 129: 47-54, 2006.

Frak V, Paulignan Y, and Jeannerod M. Orientation of the opposition axis in mentally simulated grasping. Experimental Brain Research, 136: 120-127, 2001.

Frith CD, Blakemore SJ, and Wolpert DM. Abnormalities in the awareness and control of action. Philosophical Transactions of the Royal Society of London Section B Biological Sciences, 355: 1771-1788, 2000.

Funk $\mathrm{M}$ and Brugger P. Visual recognition of hands by persons born with only one hand. Cortex, 38: 860-863, 2002.

Ganis G, Keenan JP, Kosslyn SM, and Pascual-Leone A. Transcranial magnetic stimulation of primary motor cortex affects mental rotation. Cerebral Cortex, 10: 175-180, 2000.

Gehring WJ, Himle J, and Nisenson LG. Action-monitoring dysfunction in obsessive-compulsive disorder. Psychological Science, 11: 1-6, 2000.
Genovese CR, Lazar NA, and Nichols T. Thresholding of statistical maps in functional neuroimaging using the false discovery rate. NeuroImage, 15: 870-878, 2002.

Gerardin E, Sirigu A, Lehericy S, Poline JB, Gaymard B, Marsault C, Agid Y, and Le Bihan D. Partially overlapping neural networks for real and imagined hand movements. Cerebral Cortex, 10: 1093-1104, 2000.

Glover S. Separate visual representations in the planning and control of action. Behavioural Brain Science, 27: 3-24, 2004.

Goldberg II, Harel M, and Malach R. When the brain loses its self: prefrontal inactivation during sensorimotor processing. Neuron, 50: 329-339, 2006.

Grezes J and Decety J. Functional anatomy of execution, mental simulation, observation, and verb generation of actions: a meta-analysis. Human Brain Mapping, 12: 1-19, 2001.

Grush R. The emulation theory of representation: motor control, imagery, and perception. Behavioural Brain Science, 27: 377-396, 2004.

Gusnard DA, Raichle ME, and Raichle ME. Searching for a baseline: functional imaging and the resting human brain. Nature Reviews Neuroscience, 2: 685-694, 2001.

Hajcak G and Simons RF. Error-related brain activity in obsessive-compulsive undergraduates. Psychiatry Research, 110: 63-72, 2002.

Halligan PW, Athwal BS, Oakley DA, and Frackowiak RS. Imaging hypnotic paralysis: implications for conversion hysteria. Lancet, 355: 986-987, 2000.

Hanakawa T, Immisch I, Toma K, Dimyan MA, Van Gelderen P, and Hallett $M$. Functional properties of brain areas associated with motor execution and imagery. Journal of Neurophysiology, 89: 989-1002, 2003.

Helmich RC, de Lange FP, Bloem BR, and Toni I. Cerebral compensation during motor imagery in Parkinson's disease. Neuropsychologia, 45: 2201-2215, 2007.

Jeannerod M. The representing brain: neural correlates of motor intention and imagery. Behavioural Brain Science, 17: 187-245, 1994.

Jeannerod M and Frak V. Mental imaging of motor activity in humans. Current Opinion in Neurobiology, 9: 735-739, 1999.

Jeannerod M. Motor Cognition: What Actions Tell to the Self. Oxford: Oxford University Press, 2006.

Johnson SH. Imagining the impossible: intact motor representations in hemiplegics. Neuroreport, 11: 729-732, 2000.

Johnson SH, Rotte M, Grafton ST, Hinrichs H, Gazzaniga MS, and Heinze HJ. Selective activation of a parietofrontal circuit during implicitly imagined prehension. NeuroImage, 17: 1693-1704, 2002a.

Johnson SH, Sprehn G, and Saykin AJ. Intact motor imagery in chronic upper limb hemiplegics: evidence for activityindependent action representations. Journal of Cognitive Neuroscience, 14: 841-852, 2002b.

Kawamichi H, Kikuchi Y, Noriuchi M, Senoo A, and Ueno S Distinct neural correlates underlying two- and threedimensional mental rotations using three-dimensional objects. Brain Research, 1144: 117-126, 2007.

Lacourse MG, Orr EL, Cramer SC, and Cohen MJ. Brain activation during execution and motor imagery of novel and skilled sequential hand movements. NeuroImage, 27: 505-519, 2005

Lamm C, Windischberger C, Moser E, and Bauer H. The functional role of dorso-lateral premotor cortex during mental rotation An event-related fMRI study separating cognitive processing steps using a novel task paradigm. NeuroImage, 36: 1374-1384, 2007.

de Lange FP, Kalkman JS, Bleijenberg G, Hagoort P, van der Werf SP, van der Meer JW, and Toni I. Neural correlates of the chronic fatigue syndrome - an fMRI study. Brain, 127: 1948-1957, 2004. 
de Lange FP, Hagoort P, and Toni I. Neural topography and content of movement representations. Journal of Cognitive Neuroscience, 17: 97-112, 2005.

de Lange FP, Helmich RC, and Toni I. Posture influences motor imagery: an fMRI study. NeuroImage, 33: 609-617, 2006.

de Lange FP, Roelofs $\mathrm{K}$, and Toni I. Increased self-monitoring during imagined movements in conversion paralysis. Neuropsychologia, 45: 2051-2058, 2007.

Lotze M, Grodd W, Rodden FA, Gut E, Schonle PW, Kardatzki B, and Cohen LG. Neuroimaging patterns associated with motor control in traumatic brain injury. Neurorehabilitation and Neural Repair, 20: 14-23, 2006.

Lotze M and Halsband U. Motor imagery. Journal of Physiology (Paris), 99: 386-395, 2006

Lotze M, Montoya P, Erb M, Hulsmann E, Flor H, Klose U, Birbaumer N, and Grodd W. Activation of cortical and cerebellar motor areas during executed and imagined hand movements: an fMRI study. Journal of Cognitive Neuroscience, 11: 491-501, 1999.

Marshall JC, Halligan PW, Fink GR, Wade DT, and Frackowiak RS The functional anatomy of a hysterical paralysis. Cognition, 64: B1-B8, 1997.

Maruff P and Velakoulis D. The voluntary control of motor imagery. Imagined movements in individuals with feigned motor impairment and conversion disorder. Neuropsychologia, 38: 1251-1260, 2000.

Maruff P, Wilson P, and Currie J. Abnormalities of motor imagery associated with somatic passivity phenomena in schizophrenia. Schizophrenia Research, 60: 229-238, 2003.

McFarland DJ, Miner LA, Vaughan TM, and Wolpaw JR. Mu and beta rhythm topographies during motor imagery and actual movements. Brain Topography, 12: 177-186, 2000.

Moene FC and Roelofs K. Conversion and somatisation disorder. In Nash M, and Barnier A (Eds), Oxford Handbook of Hypnosis; 2007.

Moene FC, Hoogduin KA, and Van Dyck R. The inpatient treatment of patients suffering from (motor) conversion symptoms: a description of eight cases. International Journal of Clinical and Experimental Hypnosis, 46: 171-190, 1998.

Murphy SM. Imagery interventions in sport. Medicine and Science in Sports and Exercise, 26: 486-494, 1994.

Nico D, Daprati E, Rigal F, Parsons L, and Sirigu A. Left and right hand recognition in upper limb amputees. Brain, 127: 120-132, 2004.

Owen AM, Coleman MR, Boly M, Davis MH, Laureys S, and Pickard JD. Detecting awareness in the vegetative state. Science, 313: 1402, 2006.

Parsons LM. Imagined spatial transformations of one's hands and feet. Cognitive Psychology, 19: 178-241, 1987.

Parsons LM. Temporal and kinematic properties of motor behavior reflected in mentally simulated action. Journal of Experimental Psychology: Human Perception and Performance, 20: 709-730, 1994.

Parsons LM, Gabrieli JD, Phelps EA, and Gazzaniga MS. Cerebrally lateralized mental representations of hand shape and movement. Journal of Neuroscience, 18: 6539-6548, 1998.

Pfurtscheller G, Brunner C, Schlogl A, and Lopes da Silva FH. Mu rhythm (de)synchronization and EEG single-trial classification of different motor imagery tasks. NeuroImage, 31: 153-159, 2006.

Pfurtscheller G and Neuper C. Future prospects of ERD/ERS in the context of brain-computer interface (BCI) developments. Progress in Brain Research, 159: 433-437, 2006.

Porro CA, Francescato MP, Cettolo V, Diamond ME, Baraldi P, Zuiani C, Bazzocchi M, and di Prampero PE. Primary motor and sensory cortex activation during motor performance and motor imagery: a functional magnetic resonance imaging study. Journal of Neuroscience, 16: 7688-7698, 1996.
Price CJ and Friston KJ. Degeneracy and cognitive anatomy. Trends in Cognitive Science, 6: 416-421, 2002a.

Price CJ and Friston KJ. Functional imaging studies of neuropsychological patients: applications and limitations. Neurocase, 8: 345-354, 2002b.

Pylyshyn ZW. Mental imagery: in search of a theory. Behavioural Brain Science, 25: 157-182, 2002.

Raichle ME and Mintun MA. Brain work and brain imaging. Annual Review of Neuroscience, 29: 449-476, 2006.

Richter W, Somorjai R, Summers R, Jarmasz M, Menon RS, Gati JS, Georgopoulos AP, Tegeler C, Ugurbil K, and Kim SG. Motor area activity during mental rotation studied by time-resolved single-trial fMRI. Journal of Cognitive Neuroscience, 12: 310-320, 2000.

Rodriguez M, Muniz R, Gonzalez B, and Sabate M. Hand movement distribution in the motor cortex: the influence of a concurrent task and motor imagery. NeuroImage, 22: 1480-1491, 2004.

Roelofs K, Näring GWB, Keijsers GPJ, Hoogduin CAL, Van Galen GP, and Maris E. Motor imagery in conversion paralysis. Cognitive Neuropsychiatry, 6: 21-40, 2001.

Roelofs K, De Bruijn ER, and Van Galen GP. Hyperactive action monitoring during motor-initiation in conversion paralysis: an event-related potential study. Biological Psychology, 71: 316-325, 2006.

Ruff CC, Blankenburg F, Bjoertomt O, Bestmann S, Freeman E, Haynes JD, Rees G, Josephs O, Deichmann R, and Driver J. Concurrent TMS-fMRI and psychophysics reveal frontal influences on human retinotopic visual cortex. Current Biology, 16: 1479-1488, 2006.

Rushworth MF, Johansen-Berg H, Gobel SM, and Devlin JT. The left parietal and premotor cortices: motor attention and selection. NeuroImage, 20(Suppl. 1): S89-S100, 2003.

Sauner D, Bestmann S, Siebner HR, and Rothwell JC. No evidence for a substantial involvement of primary motor hand area in handedness judgements: a transcranial magnetic stimulation study. European Journal of Neuroscience, 23: 2215-2224, 2006.

Schwoebel J, Friedman R, Duda N, and Coslett HB. Pain and the body schema: evidence for peripheral effects on mental representations of movement. Brain, 124: 2098-2104, 2001.

Sekiyama K. Kinesthetic aspects of mental representations in the identification of left and right hands. Perception and Psychophysics, 32: 89-95, 1982.

Shenton JT, Schwoebel J, and Coslett HB. Mental motor imagery and the body schema: evidence for proprioceptive dominance. Neuroscience Letters, 370: 19-24, 2004.

Shepard RN and Cooper LA. Mental Images \& Their Transformations. Cambridge, MA: MIT Press, 1982.

Sirigu A, Cohen L, Duhamel JR, Pillon B, Dubois B, Agid Y, and Pierrot-Deseilligny $C$. Congruent unilateral impairments for real and imagined hand movements. Neuroreport, 6: 997-1001, 1995.

Sirigu A and Duhamel JR. Motor and visual imagery as two complementary but neurally dissociable mental processes. Journal of Cognitive Neuroscience, 13: 910-919, 2001.

Sirigu A, Duhamel JR, Cohen L, Pillon B, Dubois B, and Agid Y. The mental representation of hand movements after parietal cortex damage. Science, 273: 1564-1568, 1996.

Spence SA, Crimlisk HL, Cope H, Ron MA, and Grasby PM. Discrete neurophysiological correlates in prefrontal cortex during hysterical and feigned disorder of movement. Lancet, 355: 1243-1244, 2000.

Stevens JA. Interference effects demonstrate distinct roles for visual and motor imagery during the mental representation of human action. Cognition, 95: 329-350, 2005.

Stone J, Carson A, and Sharpe M. Functional symptoms and signs in neurology: assessment and diagnosis. Journal of Neurology Neurosurgery and Psychiatry, 76(Suppl. 1): i2-i12, 2005. 
Strafella AP and Paus T. Cerebral blood-flow changes induced by paired-pulse transcranial magnetic stimulation of the primary motor cortex. Journal of Neurophysiology, 85: 2624-2629, 2001.

Thoenissen D, Zilles K, and Toni I. Movement preparation and motor intention: an event-related fMRI study. Journal of Neuroscience, 22: 9248-9260, 2002.

Tomasino B, Borroni P, Isaja A, and Rumiati RI. The role of the primary motor cortex in mental rotation: a TMS study. Cognitive Neuropsychology, 22: 348-363, 2005a.

Tomasino B, Budai R, Mondani M, Skrap M, and Rumiati RI. Mental rotation in a patient with an implanted electrode grid in the motor cortex. Neuroreport, 16: 1795-1800, $2005 \mathrm{~b}$.

Toni I, Schluter ND, Josephs O, Friston K, and Passingham RE. Signal-, set- and movement-related activity in the human brain: an event-related fMRI study. Cerebral Cortex, 9: 35-49, 1999.

Toni I, Thoenissen D, and Zilles K. Movement preparation and motor intention. NeuroImage, 14: S110-S117, 2001.
Ursu S, Stenger VA, Shear MK, Jones MR, and Carter CS. Overactive action monitoring in obsessive-compulsive disorder: evidence from functional magnetic resonance imaging. Psychological Science, 14: 347-353, 2003.

Vargas CD, Olivier E, Craighero L, Fadiga L, Duhamel JR, and Sirigu A. The influence of hand posture on corticospinal excitability during motor imagery: a transcranial magnetic stimulation study. Cerebral Cortex, 14: 1200-1206, 2004.

Vuilleumier P. Hysterical conversion and brain function. Progress in Brain Research, 150: 309-329, 2005.

Vuilleumier P, Chicherio C, Assal F, Schwartz S, Slosman D, and Landis T. Functional neuroanatomical correlates of hysterical sensorimotor loss. Brain, 124: 1077-1090, 2001.

Young MP, Hilgetag CC, and Scannell JW. On imputing function to structure from the behavioural effects of brain lesions. Philosophical Transactions of the Royal Society of London Section B Biological Sciences, 355: 147-161, 2000. 